\title{
Effect of celecoxib plus standard chemotherapy on serum levels of vascular endothelial growth factor and cyclooxygenase-2 in patients with gastric cancer
}

\author{
XIAOPENG HAN ${ }^{1 *}$, HONGTAO LI ${ }^{1 *}$, LIN SU ${ }^{1}$, WANKUN ZHU ${ }^{1}$, WEI XU ${ }^{1}$, \\ KUN LI $^{1}$, QINGCHUAN ZHAO ${ }^{2}, \mathrm{HUA} \mathrm{YANG}^{3}$ and HONGBIN LIU ${ }^{1}$ \\ ${ }^{1}$ Department of General Surgery, General Hospital of Lanzhou Military Region, Lanzhou, Gansu 730050; \\ ${ }^{2}$ Xijing Hospital of Digestive Diseases, The Fourth Military Medical University, Xi'an, Shaanxi 710032; \\ ${ }^{3}$ Xinqiao Hospital, The Third Military Medical University, Chongqing 400037, P.R. China
}

Received October 11,2013; Accepted November 28, 2013

DOI: 10.3892/br.2013.209

\begin{abstract}
Elevated serum levels of vascular endothelial growth factor (VEGF) and cyclooxygenase-2 (COX-2) are associated with poor prognosis in patients with gastric cancer. Little is known regarding the clinical benefits of combining celecoxib, a selective inhibitor of COX-2, with standard chemotherapy regimens for the treatment of gastric cancer patients. In this study, we investigated the effect of the combinatorial use of celecoxib with standard chemotherapy on the serum levels of VEGF and COX-2 in patients with gastric cancer. In our study, 80 patients with gastric cancer who underwent laparoscopic radical surgery were randomized into two groups, the combination [celecoxib plus standard oxaliplatin, leucovorin and 5-fluorouracil (FOLFOX4) chemotherapy, n=40] and the FOLFOX4 alone $(n=40)$ groups. In the combination group, celecoxib was orally administered to the patients $(400 \mathrm{mg}$, twice daily). The serum levels of VEGF and COX-2 were measured by ELISA prior to and following surgery. We detected no significant difference in the serum levels of VEGF and COX-2 between the combination and FOLFOX4 alone groups prior to chemotherapy $(\mathrm{P}>0.05)$. However, after 6 cycles of chemotherapy, there was a greater decrease in the serum levels of VEGF and COX-2 in the combination group compared to those in the FOLFOX4 group $(\mathrm{P}<0.01)$. In addition, the serum levels of VEGF and COX-2 were closely correlated in patients with gastric adenocarcinoma prior to treatment. Our data indicated that, when combined with standard chemotherapy, celecoxib may reduce the serum levels of VEGF and COX-2, suggesting
\end{abstract}

Correspondence to: Dr Hongbin Liu, Department of General Surgery, General Hospital of Lanzhou Military Region, 333 Binhe South Road, Lanzhou, Gansu 730050, P.R. China

E-mail: liuhongbin999@163.com

*Contributed equally

Key words: celecoxib, cyclooxygenase-2, vascular endothelial growth factor, gastric cancer, therapy that COX-2 inhibitors may be of therapeutic value through the inhibition of tumor angiogenesis and the prevention of recurrence or metastasis. Thus, celecoxib may be a useful adjuvant agent to standard chemotherapy in patients with advanced gastric cancer.

\section{Introduction}

Gastric cancer is prevalent in Gansu province in the Northwest China, particularly in the Hexi area. In Gansu province, gastric cancer has an adjusted mortality rate of 71.7 per 100,000 residents and accounts for $48.2 \%$ of all cancer-related deaths (1). In gastric cancer, the pathological stage is of prognostic significance for the 5-year survival and local recurrence. The majority of gastric cancer patients are diagnosed at an advanced stage. In these patients, tumor resection with curative intent was associated with a median survival of 24 months and the 5-year survival rate was reported to be $20-30 \%$ (2). In patients with advanced gastric cancer who underwent palliative procedures, a median survival of 8.1 months was reported, whereas without any treatment, the median survival was 5.4 months (3). Surgical resection is currently considered to be the most effective therapeutic strategy for patients with gastric cancer and more optimal clinical outcomes may be achieved if the surgical intervention is combined with addiitonal therapeutic approaches.

It was recently demonstrated that the overexpression of cyclooxygenase-2 (COX-2) and vascular endothelial growth factor (VEGF) synergistically promote local angiogenesis and lead to tumor development and metastasis (4). Thus, blocking COX-2 with its selective inhibitor celecoxib was shown to inhibit the proliferation of tumor cells and suppress the expression of VEGF, a key factor for tumor angiogenesis (5). Celecoxib was also shown to enhance the sensitivity of tumors to chemotherapy (6). For these reasons, celecoxib has been used as an adjuvant therapeutic agent for gastric cancer.

VEGF and COX-2 are detectable in $50-70 \%$ of gastric carcinomas $(7,8)$ and it was recently demonstrated that the overexpression of COX-2 and VEGF in tumor cells may synergistically promote local angiogenesis, leading to further 
Table I. Clinical parameters of enrolled patients.

\begin{tabular}{|c|c|c|c|}
\hline \multirow[b]{2}{*}{ Clinical parameters } & \multicolumn{2}{|c|}{ Groups } & \multirow[b]{2}{*}{ P-value } \\
\hline & Celecoxib + FOLFOX4 $(n=40)$ & FOLFOX4 alone $(n=40)$ & \\
\hline Gender, no. (\%) & & & $>0.05$ \\
\hline Female & $13(32.5)$ & $20(50.0)$ & \\
\hline Male & $27(67.5)$ & $20(50.0)$ & \\
\hline Median age (range) & $62(32-72)$ & $58(29-68)$ & $>0.05$ \\
\hline ECOG, no. (\%) & & & $>0.05$ \\
\hline 0 & $31(77.5)$ & $29(72.5)$ & \\
\hline 1 & $9(22.5)$ & $11(27.5)$ & \\
\hline Tumor pathology, no. (\%) & & & $>0.05$ \\
\hline Well differentiated & $9(22.5)$ & $7(17.5)$ & \\
\hline Moderately differentiated & $9(22.5)$ & $10(25.0)$ & \\
\hline Poorly differentiated & $19(47.5)$ & $21(52.5)$ & \\
\hline Mucinous & $3(7.5)$ & $2(5.0)$ & \\
\hline Clinical stage, no. (\%) & & & $>0.05$ \\
\hline I & $5(12.5)$ & $4(10.0)$ & \\
\hline II & $22(55.0)$ & $19(47.5)$ & \\
\hline III & $10(25.0)$ & $15(37.5)$ & \\
\hline $\mathrm{V}$ & $3(7.5)$ & $2(5.0)$ & \\
\hline
\end{tabular}

FOLFOX4, oxaliplatin, leucovorin and 5-fluorouracil; ECOG, Eastern Cooperative Oncology Group.

tumor development and metastasis (4). VEGF is one of the most potent angiogenic factors and is crucial for the persistent proliferation and metastasis of tumor cells (9). The expression of VEGF and COX-2 is closely associated with clinical stage, lymph node metastasis and prognosis of gastric cancer, suggesting that these factors play important roles in the development of gastric cancer (10). Furthermore, high expression levels of VEGF $(8,10)$ and COX-2 $(7,8)$ were shown to be associated with reduced disease-free survival and increased mortality rate over a 5-year period in patients with gastric cancer. The close association between VEGF and COX-2 and poor prognosis suggest that these two molecules may be used as biomarkers for disease progression, as well as potential therapeutic targets.

Multiple in vitro and animal studies indicated that celecoxib may inhibit the growth of gastric tumors and suppress tumor angiogenesis by sensitizing tumor cells to chemotherapy-induced apoptosis $(11,12)$. Celecoxib was demonstrated to be highly potent and relatively non-toxic in animal tumor models. Moreover, it significantly decreased the production of cytokines associated with gastric tumor development and metastasis. The synergistic effects of celecoxib and 5-fluorouracil, cisplatin, or etoposide were previously demonstrated in the BGC-823 gastric cancer cell line (13). Similarly, celecoxib was shown to synergistically inhibit tumor growth, angiogenesis and metastasis in patients with esophageal cancer when combined with other chemotherapeutic agents (14). However, the clinical value of celecoxib in the management of patients with gastric cancer has not been clearly determined.

In order to elucidate the clinical usefulness of VEGF and COX-2 in the management of patients with advanced gastric cancer, we measured the serum levels of VEGF and COX-2 in gastric cancer patients who received celecoxib in combination with the standard chemotherapy regimen of oxaliplatin, leucovorin and 5-fluorouracil (FOLFOX4) following laparoscopic radical gastrectomy.

\section{Materials and methods}

Patient selection and characteristics. A total of 80 patients with gastric cancer, including 48 men and 32 women aged 18-70 years, who underwent laparoscopic radical surgery for gastric cancer between 2010 and 2012 were enrolled in this study. The patients were randomly assigned to two groups, the celecoxib plus FOLFOX4 $(n=40)$ and the FOLFOX4 alone $(n=40)$ groups. None of the enrolled patients had received previous chemotherapy. The ECOG performance status score range was 0-2, indicating no dysfunction of vital organ systems. The functional tests of the major organ systems (heart, liver, kidney, blood and lung) revealed no obvious abnormalities and all the patients had normal blood cell counts.

This study was approved by the Ethics Committee of the General Hospital of Lanzhou Military Region (Lanzhou, China) and all the patients provided written informed consent prior to enrollment.

Patient exclusion criteria. The patients were excluded from this study if they exhibited one or more of the following: a severe allergic history; uncontrolled diabetes mellitus; uncontrolled hypertension; active peptic ulcer; any severe condition or dysfunction affecting the heart, lung, liver, kidney, blood, 
Table II. Serum levels of VEGF and COX-2 following treatment with or without celecoxib.

\begin{tabular}{|c|c|c|c|c|c|c|c|c|}
\hline \multirow[b]{3}{*}{ Markers } & \multirow{2}{*}{\multicolumn{2}{|c|}{ Pre-surgery }} & \multicolumn{6}{|c|}{ Post-surgery } \\
\hline & & & \multicolumn{2}{|c|}{3 weeks } & \multicolumn{2}{|c|}{3 months } & \multicolumn{2}{|c|}{5 months } \\
\hline & $\begin{array}{c}\mathrm{pg} / \mathrm{ml} \\
(\text { mean } \pm \mathrm{SD})\end{array}$ & t/P-value & $\begin{array}{c}\mathrm{pg} / \mathrm{ml} \\
(\mathrm{mean} \pm \mathrm{SD})\end{array}$ & $\mathrm{t} / \mathrm{P}$-value & $\begin{array}{c}\mathrm{pg} / \mathrm{ml} \\
(\mathrm{mean} \pm \mathrm{SD})\end{array}$ & t/P-value & $\begin{array}{c}\mathrm{pg} / \mathrm{ml} \\
(\text { mean } \pm \mathrm{SD})\end{array}$ & t/P-value \\
\hline \multicolumn{9}{|l|}{ VEGF } \\
\hline $\begin{array}{l}\text { Celecoxib + } \\
\text { FOLFOX4 }\end{array}$ & $405.5 \pm 83.2^{\mathrm{a}}$ & $t=1.64$ & $401.5 \pm 81.1$ & $\mathrm{t}=1.43$ & $265.3 \pm 54.9$ & $t=4.02$ & $212.5 \pm 52.2$ & $\mathrm{t}=3.45$ \\
\hline FOLFOX4 & $442.9 \pm 78.3^{\mathrm{a}}$ & $\mathrm{P}=0.11$ & $434.9 \pm 82.9$ & $\mathrm{P}=0.15$ & $325.1 \pm 50.2$ & $\mathrm{P}<0.01$ & $266.3 \pm 57.7$ & $\mathrm{P}<0.01$ \\
\hline Controls & $117.2 \pm 20.1$ & $\mathrm{P}<0.01$ & - & - & - & - & - & - \\
\hline \multicolumn{9}{|l|}{ COX-2 } \\
\hline $\begin{array}{l}\text { Celecoxib + } \\
\text { FOLFOX4 }\end{array}$ & $36.5 \pm 10.8^{a}$ & $\mathrm{t}=1.88$ & $36.1 \pm 10.8$ & $\mathrm{t}=1.48$ & $24.9 \pm 4.9$ & $\mathrm{t}=5.79$ & $19.3 \pm 5.7$ & $\mathrm{t}=4.29$ \\
\hline FOLFOX4 & $41.9 \pm 9.5^{\mathrm{a}}$ & $\mathrm{P}=0.06$ & $40.3 \pm 9.3$ & $\mathrm{P}=0.14$ & $33.2 \pm 5.3$ & $\mathrm{P}<0.001$ & $25.6 \pm 5.7$ & $\mathrm{P}<0.001$ \\
\hline Controls & $16.9 \pm 6.3$ & $\mathrm{P}<0.001$ & - & - & - & - & - & - \\
\hline
\end{tabular}

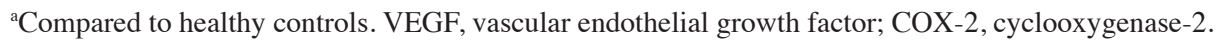

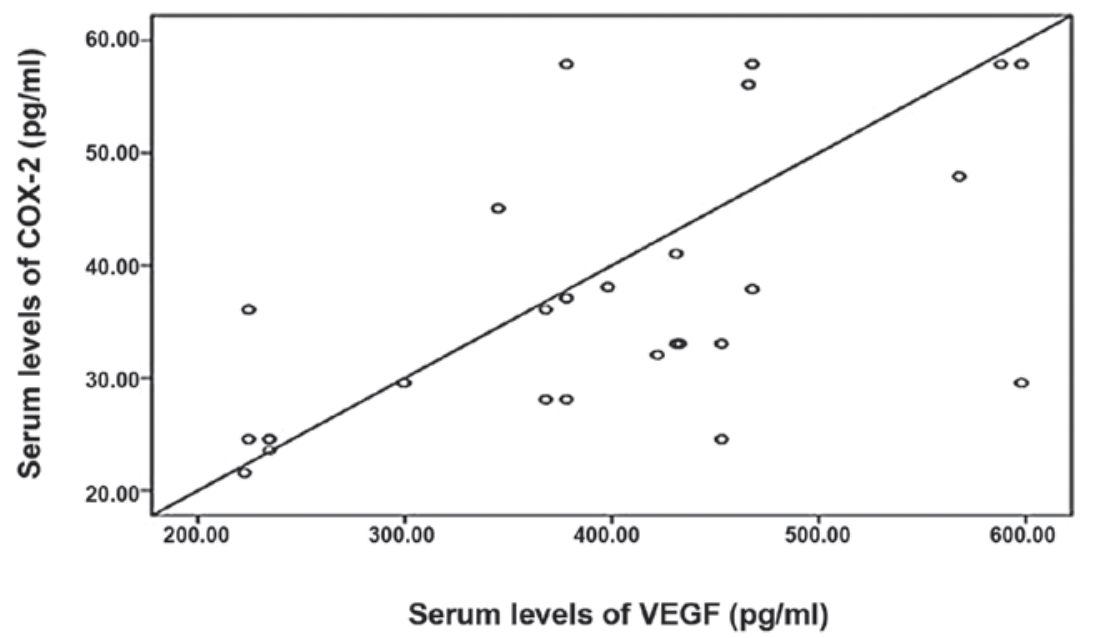

Figure 1. Association between the serum levels of vascular endothelial growth factor (VEGF) and cyclooxygenase-2 (COX-2). The fasting serum levels of VEGF and COX-2 were measured by ELISA in gastric cancer patients prior to chemotherapy. A strong correlation between VEGF and COX-2 is observed.

or bone marrow; a history of uncontrolled mental disorders; pregnant or nursing women; and symptoms of brain metastasis.

Furthermore, the patients were excluded from this study if they had received: other chemotherapy or molecular-targeted therapy; non-standard FOLFOX4 chemotherapy (see below); or standard FOLFOX4 chemotherapy, but the treatment was discontinued due to intolerable adverse effects or disease exacerbation.

Treatment regimens. The standard FOLFOX4 chemotherapy regimen consists of oxaliplatin $\left(85 \mathrm{mg} / \mathrm{m}^{2}\right.$, intravenous, day 1$)$, leucovorin $\left(200 \mathrm{mg} / \mathrm{m}^{2}\right.$, intravenous, days 1 and 2 , over $2 \mathrm{~h}$ ) and 5-fluorouracil (400 mg/m², intravenous, over $22 \mathrm{~h}$ ). The cycle was repeated every 4 weeks and the patients received a total of 6 cycles. In patients with combination therapy, in addition to the above-described FOLFOX4 regimen, celecoxib (Pfizer, New York, NY, USA) was administered at a dose of
$400 \mathrm{mg}$ orally twice a day for 5 months to the completion of the chemotherapy.

Chemotherapy-related side-effects were managed by standard protocols, including boosting white blood cell and platelet counts, use of antiemetics and protection of liver function. Other therapeutic approaches, such as radiotherapy and traditional Chinese herbal medicines, were not used during the treatment cycles. All the patients were followed up through regular visits to the outpatient department and telephone reviews.

Detection of serum levels of VEGF and COX-2. Fasting blood samples $(3 \mathrm{ml})$ were collected from each patient prior to and 3 weeks after surgery, prior to chemotherapy and after 3 and six cycles of chemotherapy. Blood samples were also collected from 30 healthy subjects and were used as controls. Written consent was obtained from all the healthy subjects. The serum was separated by centrifugation at $1358.37 \mathrm{x} g$ for 
$10 \mathrm{~min}$ at $4^{\circ} \mathrm{C}$ and stored at $-80^{\circ} \mathrm{C}$ for further analysis. The concentrations of VEGF and COX-2 were determined by the respective ELISA kits (LifeKey Research, Scottsdale, AZ, USA) according to the manufacturer's protocols. The optical density (OD) was read in triplicate and the average OD reading was used to quantify VEGF and COX-2 concentration from the standard curve provided.

Statistical analysis. Statistical analysis was conducted using the SPSS 16 software (SPSS Inc., Chicago, IL, USA). The data were analyzed by a t-test and the correlation analysis was conducted by the linear regression method. $\mathrm{P}<0.05$ was considered to indicate a statistically significant difference.

\section{Results}

Clinical parameters. No significant difference was observed in the baseline clinical parameters between patients receiving the combination therapy (i.e., celecoxib + FOLFOX4) and FOLFOX alone $(\mathrm{P}>0.05)$ (Table I).

Serum levels of VEGF and COX-2. Significantly higher levels of VEGF and COX-2 were found in patients with gastric cancer compared to those in healthy control subjects $(\mathrm{P}<0.01)$. No significant difference was observed in the serum levels of VEGF and COX-2 between patients receiving combination therapy and those treated with FOLFOX4 alone, either prior to or 3 weeks after the surgery and prior to chemotherapy (Table II) $(\mathrm{P}>0.05)$. However, a greater reduction in the serum levels of VEGF and COX-2 was observed in patients who received combination therapy compared to those treated with 6 cycles of FOLFOX4 alone (Table II) $(\mathrm{P}<0.01)$.

Correlation between serum levels of VEGF and COX-2. A significant positive correlation between the serum levels of VEGF and COX-2 was observed in patients with gastric cancer prior to treatment, as revealed by the Pearson's correlation analysis (Fig. 1, $\mathrm{R}=0.631, \mathrm{P}<0.05$ ).

\section{Discussion}

Gastric cancer is the fourth most common malignant tumor and the second most common cause of cancer-related mortality worldwide. Although the incidence of gastric cancer is continuously decreasing in Western countries, it remains high in Asia, Latin America and Eastern Europe (3). Surgery remains the mainstay of treatment; however, a recurrence rate of $\leq 40 \%$ following radical gastrectomy has been reported (15). Chemotherapy, such as the FOLFOX4 regimen, following surgery in patients with intermediate or advanced gastric cancer may reduce the recurrence rate of gastric cancer and improve patient survival (16). Celecoxib, a selective COX-2 inhibitor, may provide an additional benefit by suppressing angiogenesis and inducing tumor cell apoptosis, thereby improving the treatment outcome (4). Furthermore, celecoxib is relatively non-toxic and, when used in combination with other chemotherapeutic agents, it may enhance the sensitivity of the tumor cells to chemotherapeutic drugs, thereby magnifying the therapeutic benefits $(5,17)$. Thus, in patients with gastric cancer, the combinatorial application of celecoxib with other chemotherapeutic agents may improve the treatment outcome without causing intolerable toxicity.

In the present study, we observed that, when celecoxib was used in combination with standard FOLFOX4 chemotherapy in patients with advanced gastric cancer, there was a gradual reduction in the serum levels of VEGF and COX-2 (two commonly used serum biomarkers for tumor recurrence), as opposed to patients treated with FOLFOX4 alone, suggesting an additional benefit of the COX-2 inhibition in the management of patients with advanced gastric cancer.

The high expression levels of VEGF and COX-2 in gastric tissues and in the serum of patients with gastric cancer may contribute to gastric cancer progression (10). As VEGF is a potent angiogenic factor, enhancing tumor angiogenesis and contributing to tumor progression and metastasis, and COX-2 has been reported to regulate the expression and function of VEGF in cancers, the observed close correlation between the serum levels of VEGF and COX-2 indicates that blocking COX-2 may help reduce the neovascularization required for tumor growth. This observation is consistent with the findings of previous studies (17). As such, the serum levels of VEGF and COX-2 may serve as valuable biomarkers for the assessment of therapeutic benefit in gastric cancer patients receiving anticancer therapy.

Larger clinical studies are required to further evaluate the association between VEGF and COX-2 as markers of disease progression in gastric cancer patients prior to and after therapy. The value of the serum levels of VEGF and COX-2 in determining the prognosis of patients with gastric cancer also requires further investigation. Finally, clinical trials should be performed, involving larger patient cohorts and conducting short- and long-term follow-up, in order to determine whether celecoxib affects patient survival and quality of life and assess its possible side-effects in gastric cancer patients.

\section{References}

1. Zhou Y, Zhang Z, Zhang Z, et al: A rising trend of gastric cardia cancer in Gansu Province of China. Cancer Lett 269: 18-25, 2008.

2. Qin X and Liu FL: Efforts to improve postoperative 5-year survival rate of gastric cancer. Journal of Surgery Concepts and Practice 13: 4-6, 2008 (In Chinese).

3. Dicken BJ, Bigam DL, Cass C, et al: Gastric adenocarcinoma: review and considerations for future directions. Ann Surg 241: 27-39, 2005.

4. Yao L, Liu F, Hong L, et al: The function and mechanism of COX-2 in angiogenesis of gastric cancer cells. J Exp Clin Cancer Res 30: 13, 2011

5. Liu H, Huang P, Xu X, et al: Anticancer effect of celecoxib via COX-2 dependent and independent mechanisms in human gastric cancers cells. Dig Dis Sci 54: 1418-1424, 2009.

6. Zhang DQ, Guo Q, Zhu JH, et al: Increase of cyclooxygenase-2 inhibition with celecoxib combined with 5-FU enhances tumor cell apoptosis and antitumor efficacy in a subcutaneous implantation tumor model of human colon cancer. Word J Surg Oncol 11: 16, 2013

7. Gou HF, Chen XC, Zhu J, et al: Expression of COX-2 and VEGF-C in gastric cancer: correlations with lymphangiogenesis and prognostic implications. J Exp Clin Cancer Res 30: 14, 2011.

8. Da MX, Wu XT, Wang J, et al: Expression of cyclooxygenase-2 and vascular endothelial growth factor-C correlates with lymphangiogenesis and lymphatic invasion in human gastric cancer. Arch Med Res 39: 92-99, 2008.

9. Leung WK, To KF, Go MY, et al: Cyclooxygenase-2 upregulates vascular endothelial growth factor expression and angiogenesis in human gastric carcinoma. Int J Oncol 23: 1317-1322, 2003. 
10. Kolev Y, Uetake H, Iida S, et al: Prognostic significance of VEGF expression in correlation with COX-2, microvessel density, and clinicopathological characteristics in human gastric carcinoma. Ann Surg Oncol 14: 2738-2747, 2007.

11. Kuo CH,Hu HM, Tsai PY, et al: Short-term celecoxib intervention is a safe and effective chemopreventive for gastric carcinogenesis based on a Mongolian gerbil model. World J Gastroenterol 15 4907-4914, 2009.

12. Hu PJ, Yu J, Zeng ZR, et al: Chemoprevention of gastric cancer by celecoxib in rats. Gut 53: 195-200, 2004.

13. Zhu FS, Chen XM, Wang YJ, Zhang X and Feng JX: Antitumor effects of specific cyclooxygenase inhibitors combined with chemotherapeutic agents on gastric cancer cells in vitro. Chin J Oncol 29: 186-188, 2007 (In Chinese).
14. Allegrini G, Di Desidero T, Barletta MT, et al: Clinical, pharmacokinetic and pharmacodynamic evaluations of metronomic UFT and cyclophosphamide plus celecoxib in patients with advanced refractory gastrointestinal cancers. Angiogenesis 15: 275-286, 2012.

15. Du X, Nie H, Liang Y, et al: The effect of chemotherapy treatment on $\mathrm{T}$ cell subgroups, NK cell in patients after radical gastrectomy. Hebei Med J 32: 49-50, 2011.

16. Yoshida K, Yamaguchi K, Osada S, et al: Challenge for a better combination with basic evidence. Int J Clin Oncol 13: 212-219, 2008.

17. Zhou Y, Ran J, Tang C, et al: Effect of celecoxib on E-cadherin, VEGF, microvessel density and apoptosis in gastric cancer. Cancer Biol Ther 6: 269-275, 2007. 